\title{
XRCC2 Is Required for the Formation of Rad51 Foci Induced by lonizing Radiation and DNA Cross-Linking Agent Mitomycin C
}

\author{
Nan Liu* \\ Biology and Biotechnology Research Program, Lawrence Livermore National Laboratory, Livermore, CA 94551, USA
}

Received 28 February 2002; accepted 28 April 2002

\begin{abstract}
XRCC2 protein shares weak amino acid sequence similarity with Rad51, which is a central player in homologous recombinational repair (HRR). Rad51 proteins assemble at the sites of HRR and form visible nuclear foci in response to DNA damage. Xrcc2 hamster mutant irs1 cells are incapable of forming Rad51 foci after ionizing irradiation or DNA cross-linking agent mitomycin C treatment, though the Rad51 protein level is normal in the mutant. The defect can be corrected in an XRCC2 transformant. Time course study showed that the irs 1 cells primarily lacked the early response ( 2 hours after irradiation) to form small Rad51 foci (type 1 ) and later response ( 8 hours after irradiation) to form large foci (type 2). These results suggested that XRCC2 is essential for the assembly of the DNA damage-induced Rad51 foci and that XRCC2 may play an important role in the early stage of HRR.
\end{abstract}

\section{INTRODUCTION}

Xrcc2 mutant irs1 cell line is hypersensitive to various DNA damaging agents such as ionizing radiation, UV, alkylating agents, and cross-linking agents [6, 19]. Irs1 cells also show elevated levels of spontaneous or DNA damageinduced chromosomal aberrations and chromosomal rearrangement $[9,21,45]$. The human XRCC2 gene was isolated by a functional complementation in irs 1 cells, and the predicted amino acid sequence of XRCC2 protein revealed a similarity to Rad51 [7, 21]. XRCC2 belongs to a family of Rad51 paralogs, which includes XRCC2, XRCC3, Rad51B (HsRec2 or Rad51L1), Rad51C, and Rad51D (Rad51L3). All of the Rad51 paralogs share marginal sequence similarity (20-30\%) with Rad51 and are present specifically in vertebrates. XRCC 3 is also isolated by functional complementation in $x r c c 3$ hamster mutant irs1SF $[21,43]$ whose phenotype is markedly similar to that of irs1 [11]. $\operatorname{Rad} 51 \mathrm{~B}, \mathrm{C}$, and $\mathrm{D}$ are identified in the database by searching for protein sequences homologous to Rad51 (reviewed in [44]). Using a recombination reporter system, it has been demonstrated that repair of site-specific DNA double strand break (DSB) mediated by homologous recombination is dramatically (100-fold) reduced in irs1 cells compared to the wild type [17]. Irs1 cells are also deficient in repair of DSBs that are induced as intermediate products in repair of DNA cross-linking damage [10]. These lines of evidence suggest that XRCC2 plays an important role in homologous recombinational repair (HRR) of DSB. However, the mechanism underlying its function is still not clear.
DNA double strand breaks are highly genotoxic lesions that can lead to chromosomal instability and mutagenesis if they are not repaired accurately. In mammalian cells, DSBs are repaired by two major pathways, the error-prone nonhomologous end-joining and the error-free homologous recombination. It is known that in yeast $S$ cerevisiae, the proteins in Rad52 epistasis group (RAD50, RAD51, RAD52, RAD54, RAD55, RAD57, RAD59, RDH54/TID1, MRE11, and $X R S 2)$ are responsible for repair of DSBs occurred in meiosis or induced by ionizing radiation. Among these proteins, Rad51 plays a central role in HRR. Rad51 is a homolog of $E$ coli RecA recombinase and its structure and function are highly conserved in mammals. Biochemical studies showed that Rad51 proteins form nucleoprotein filaments on singlestranded DNA in the presynaptic stage of HRR and mediate homologous pairing and strand exchange between singlestranded DNA and homologous double-stranded DNA [1, 14, 35]. Rad51 interacts with Rad52 and Rad54, which both promote the strand paring and exchange by Rad51 (reviewed in [38]). The yeast Rad55 and Rad57 proteins share remote sequence similarity to Rad51 and form a heterodimer that stimulates the activity of Rad51 [36]. In yeast and eukaryotic cells, Rad51 proteins form discrete nuclear foci following the induction of DSBs in meiosis or in mitotic cells treated with ionizing radiation or other DNA damaging agents $[3,15]$. The biological significance of Rad51 foci is emphasized by the finding that many of the proteins involved in HRR, such as RPA (replication protein A), Rad52, and Rad54, are not only interact with Rad51, but also colocalize with Rad51 foci $[23,32,33,41]$. In fact, Rad52, Rad54, and Rad55-Rad57 are required for the cellular response of Rad51 focus formation 
$[13,41]$. The breast cancer suppressor proteins, BRCA1 and BRCA2, directly or indirectly interact with $\operatorname{Rad} 51[8,31]$. These proteins are also required for Rad51 focus formation $[2,47]$ and their DNA damage-induced foci colocalize with $\operatorname{Rad} 51$ foci $[8,31]$. Moreover, recent studies have shown that all of the Rad51 paralogs are essential for the formation of Rad51 foci $[4,29,39,40]$.

In this paper, I report the finding that Rad51 focus formation is defective in $x r c c 2$ hamster mutant irs 1 cells after treatment with ionizing radiation and cross-linking agent mitomycin C (MMC). The results suggest that XRCC2 plays a role as a mediator to promote the activity of Rad51 in homologous recombinational repair.

\section{MATERIALS AND METHODS}

\section{Cell lines}

The human HeLa I and Chinese hamster cell lines V79, its derived mutant irs1, and the XRCC2 transformants of irs1 (pDXR2 and GT621) were cultured in monolayers in $\alpha$ MEM medium supplemented with $10 \%$ fetal bovine serum and antibiotics as described in [21]. The cells were grown at $37^{\circ} \mathrm{C}$ in a humidified $7 \% \mathrm{CO}_{2}$ atmosphere.

\section{$\gamma$-irradiation and MMC treatment}

Cells were seeded in chamber slides and grown at $37^{\circ} \mathrm{C}$ overnight. Cells were then irradiated with ${ }^{137} \mathrm{Cs} \gamma$-rays at room temperature for $8 \mathrm{~Gy}$ at $1.83 \mathrm{~Gy} / \mathrm{min}$. After irradiation, cells were taken back to $37^{\circ} \mathrm{C}$ immediately and fixed after incubation for various period of time (30 minutes to 24 hours). For MMC treatment, cells were incubated with MMC at various concentrations (0-200 nM) in the chamber for 16 hours before being fixed.

\section{Immunostaining}

The cells grown on the chamber slides were washed 3 times with phosphate-buffered saline (PBS) and fixed with $1 \%$ paraformadehyde in PBS for 15 minutes at room temperature. Cells were permeabalized with methanol-aceton (1 : 1) on ice for 1 minute. Slides were blocked with $1 \%$ bovine serum albumin (BSA) for 1 hour, incubated with rabbit antimouse Rad51 antibody $(\alpha$-MmRad51) in $1 \%$ BSA for 1 hour, and incubated with fluorescein isothiocyanate (FITC)-conjugated antirabbit IgG (Amersham, Piscataway, NJ, USA) for 1 hour. Slides were extensively washed with PBS following each incubation. Cover slices were mounted onto the slides with Vectashield mounting medium (Vector laboratories Inc, Burlingame, Calif, USA) containing DAPI $(0.1 \mathrm{~g} / \mathrm{mL})$. Immunostained slides were examined under a fluorescent microscope using a $1000 \times$ Ziess objective, and digital images were taken and recorded using software Pathvysion (Applied Imaging, San Jose, Calif, USA). At least 200 nuclei were scored and a threshold of 5 foci/cell was used.

\section{RESULTS AND DISCUSSION}

The hamster irs1 mutant cell line is effectively XRCC2 null due to an early frameshift truncation mutation [21].
Expression of human XRCC2 partially or fully corrected the hypersensitivity of irs 1 to ionizing radiation, mitomycin $\mathrm{C}$, and cisplatin [21]. Since XRCC2 protein shares low level of sequence similarity to $\operatorname{Rad} 51[7,21]$ and is involved in DSB repair [17], it is suggested that XRCC2 may act as a cofactor of Rad51. However, the function of XRCC2 in Rad51mediated HRR is still unknown. To investigate the functional link of XRCC2 with Rad51, the formation of Rad51 foci is examined in $x r c c 2$ hamster mutant irs1. Cells were irradiated with $8 \mathrm{~Gy} \gamma$-rays and incubated at $37^{\circ} \mathrm{C}$ for 2 hours, and then were fixed and immunostained with MmRad51 antibody. In wild-type V79 cells, Rad51 foci were readily detected in nuclei 2 hours after irradiation (Figure 1a). In contrast, the response of forming Rad51 foci is diminished in irradiated irs 1 cells (Figure 1a). Rad51 foci are restored in an XRCC2 genomic DNA (phage artificial chromosome (PAC) clone) transformant GT621 to the wild-type level, but are not fully restored in an XRCC2 cDNA transformant pDXR2 (see Figure 1b). A previous study has shown that these transformants exhibit different degrees of correction for cell survival after ionizing irradiation and MMC treatment [21]. The survival of GT621 was rescued to a wild-type level after $\gamma$ irradiation and MMC treatment, while pDXR2 showed partial correction for MMC and little correction for $\gamma$-ray irradiation. The partial correction in pDXR2 cells may be resulted from an abnormal level of XRCC2 expression [21].

The frequencies of Rad51 focus-positive cells before and after irradiation are shown in Figure 1b. Before irradiation, the percentage of cells containing Rad51 foci shows no difference between V79 and irs1 cells, though the ratio $(<2 \%)$ is much lower than that in HeLa I cells (12.6\%) (Figure 1b). Since the Rad51 foci seen in unirradiated cells are primarily formed in the S phase [42], this result suggests that the $S$ phase Rad51 foci formation may not be severely affected in irs 1 mutant. Two hours after irradiation, the percentage of cells containing the foci increased markedly in V79 cells (59.0\%), as well as in HeLa I cells (50.7\%). In addition, the number of Rad51 nuclear foci per cell is also increased in V79 and HeLa I cells after ionizing irradiation (data not shown). In contrast, irs 1 cells showed no such response and the number of cells containing Rad51 foci showed little increase (Figure 1b). This result is consistent with the data reported recently, which also showed that the irs1 lacked the capability to form Rad51 foci after irradiation [29]. In GT621 cells, Rad51 focus formation was fully restored (70.9\%) after irradiation (Figure 1b). However, only a slight increase of Rad51 foci was found in pDXR2 cells (14.3\%) (Figure 1b). These results suggest that the response of Rad51 focus formation to DNA damage correlates well with the cellular sensitivity to DNA damaging agents.

Irs 1 cells are extremely sensitive to DNA cross-linking agents, such as MMC, cisplatin, and nitrogen mustard. The increased killing of irs1 by MMC is partially corrected in pDXR2 and fully corrected in GT621 [21]. To examine the Rad51 focus formation, the cells were incubated at $37^{\circ} \mathrm{C}$ at various concentrations of MMC for 16 hours. In HeLa I cells, the Rad51 focus-positive cells increase after treatment with MMC in a dose-dependent manner and reached to a plateau 

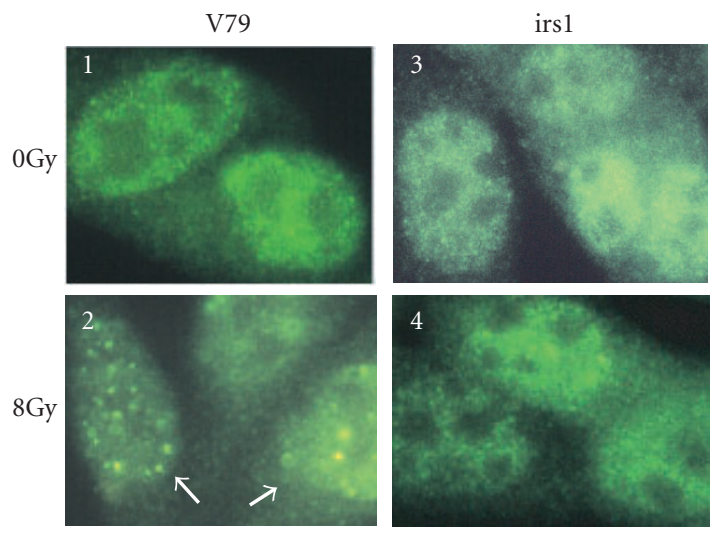

(a)

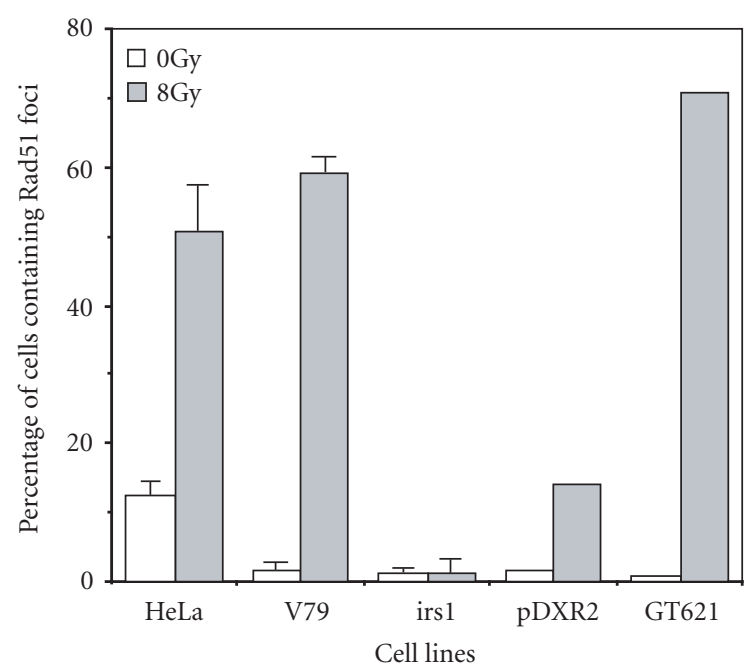

(b)

Figure 1. Rad51 focus formation induced by $\gamma$-ray irradiation. (a) Immunostaining with mouse $\alpha$-Rad51 antibody of wild-type V79 and $x r c c 2$ mutant irs 1 nuclei 2 hours after 8 Gy $\gamma$-irradiation. The nuclei containing Rad51 foci are indicated with arrows. (b) Percentage of cells containing Rad51 foci before and after $\gamma$-ray irradiation in HeLa, V79, irs1, XRCC2 cDNA transformant pDXR2, and genomic transformant GT621. At least 200 nuclei for each sample were scored in each experiment and a threshold of 5 foci/cell was used. Error bars indicate the standard deviations of the average values from two independent experiments. One experiment was done for pDXR2 and GT621.

at $100 \mathrm{nM}$ (Figure 2). The foci induced by MMC appeared to be smaller and less intensive than the foci induced by $\gamma$-rays. The number of cells containing Rad51 foci also increased markedly in V79 and GT621 cells after MMC treatment (Figure 2). However, the positive cells are greatly reduced in irs 1 and pDXR2 cells at all dose points tested, compared to the wild type (Figure 2), though the Rad51 focuspositive cells increased slightly after continuous exposure to MMC (Figure 2).

To rule out the possibility that the diminished Rad51 focus formation in irs 1 cells is due to reduced Rad51 protein

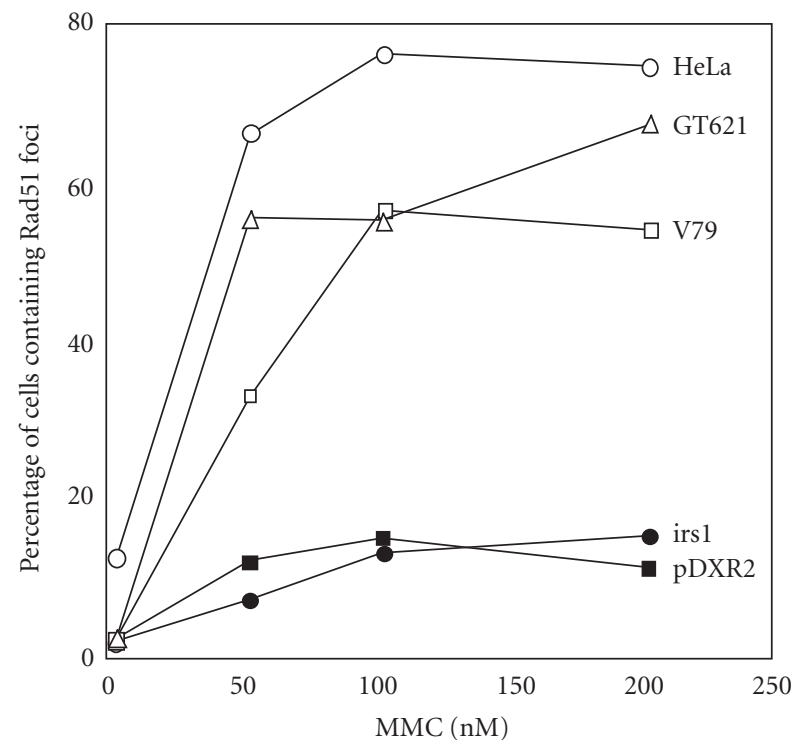

Figure 2. Induction of Rad51 nuclear foci by MMC treatment. HeLa, V79, irs1, pDXR2, and GT621 cells were incubated with MMC at various concentrations at $37^{\circ} \mathrm{C}$ for 16 hours.

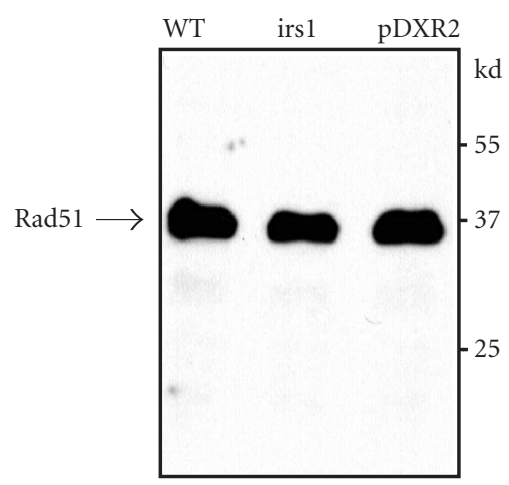

Figure 3. Western blot with $\alpha$-MmRad51 antibody. Cell extracts $(40 \mu \mathrm{g})$ of V79, irs1, or pDXR2 was loaded and proteins were seperated on 10\% SDSPAGE gel. The hamster Rad51 (37 kd) is indicated.

level, Western blotting with $\alpha$-MmRad51 antibody was performed to determine the Rad51 protein in V79, irs1, and pDXR2. Hamster Rad51 was readily detected in all cell lines and the expression levels showed no difference among V79, irs1, and pDXR2 cells (Figure 3). In addition, several studies showed that the Rad51 protein expression is not induced by ionizing radiation [4, 47], so the Rad51 level in irradiated cells should remain unchanged. This result suggests that the defect of Rad51 focus formation in irs1 is not caused by reduced Rad51 protein level, but may lie in the redistribution or assembly of Rad51 after the DNA damage. Rad51 protein level appeared to be normal in the absence of XRCC2, indicating that XRCC2 may not influence the stability of Rad51.

A recent study showed that $\operatorname{Rad} 51$ foci are induced by ionizing radiation during the $S$ phase but not in G1 [12]. To ascertain whether the Rad51 focus formation is delayed in 
Time after $\gamma$-ray irradiation

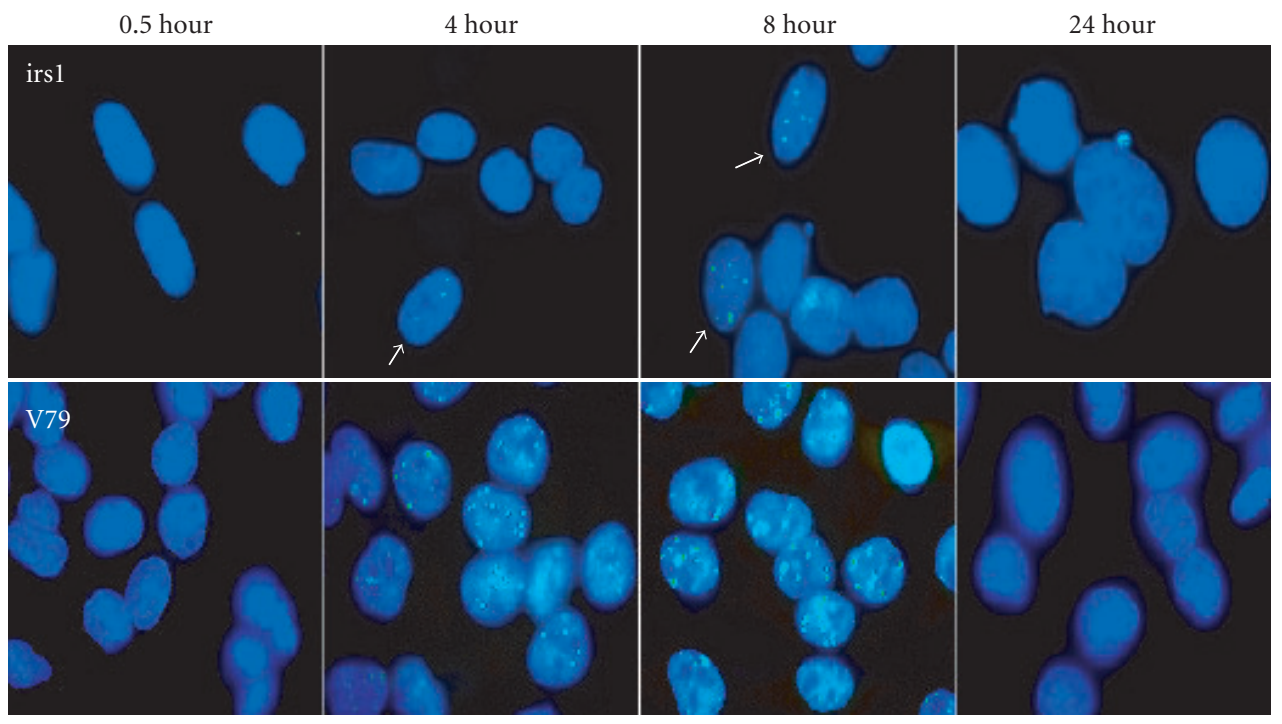

(a)

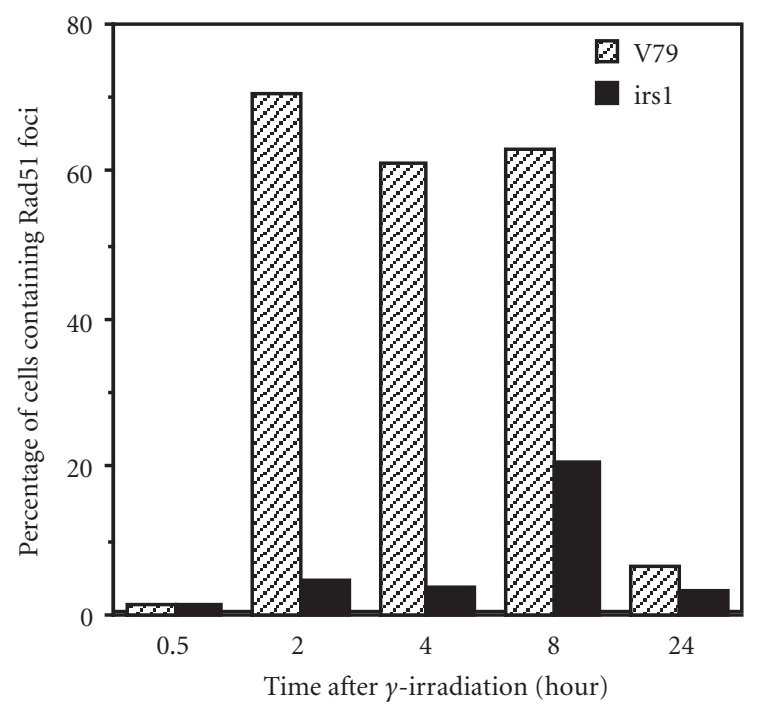

(b)

Figure 4. Time course of Rad51 focus formation. (a) Combined image of FITC (Rad51) and DAPI (nuclei) staining at 0.5, 4, 8, and 24 hours after irradiation. Irs1 (top panels) and V79 (bottom panels) cells were irradiated with $8 \mathrm{~Gy} \gamma$-rays and incubated at $37^{\circ} \mathrm{C}$ for various times. The irs 1 nuclei contain the small foci (type I foci) are indicated with arrows. (b) Percentage of cells containing Rad51 foci at the time points after irradiation.

irs1 cells because of ionizing radiation-caused or mutagencaused cell cycle delay, the time course of Rad51 focus formation was examined in V79 and irs1 cells. Cells were irradiated with $8 \mathrm{~Gy} \gamma$-rays and then incubated at $37^{\circ} \mathrm{C}$ for various times. Very few foci was seen in V79 or irs 1 cells 30 minutes after 8 Gy irradiation (Figure 4). In V79 cells, most of the cells containing the foci appeared at 2 hours and disappeared at 24 hours after irradiation (Figures $4 \mathrm{a}$ and $4 \mathrm{~b}$ ). The formation of the foci is significantly reduced in irs1 at all time points compared to the wild-type V79 cells (Figures 4a and $4 \mathrm{~b})$. However, more irs 1 cells showed Rad51 foci at 8 hours compared to irs 1 cells harvested at other time points. It is noticed that the foci appeared in irs 1 cells 8 hours after irradiation are exclusively small foci (Figure 4a). In general, the Rad51 foci could be viewed as two types in terms of the size of the foci, the small foci (type I) and the large foci (type II) (Figure 5a). In V79 cells 2 hours after irradiation, the majority of cells contains only type I foci, while cells containing 


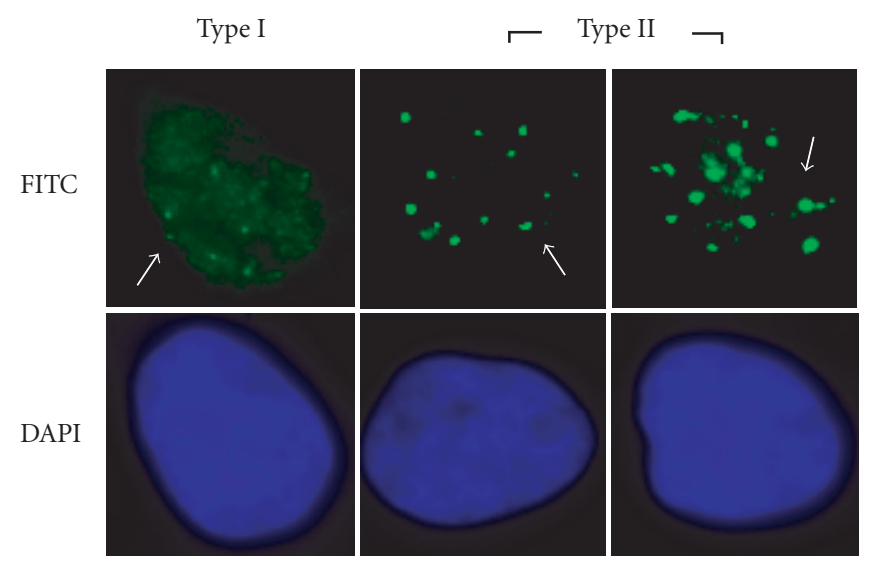

(a)
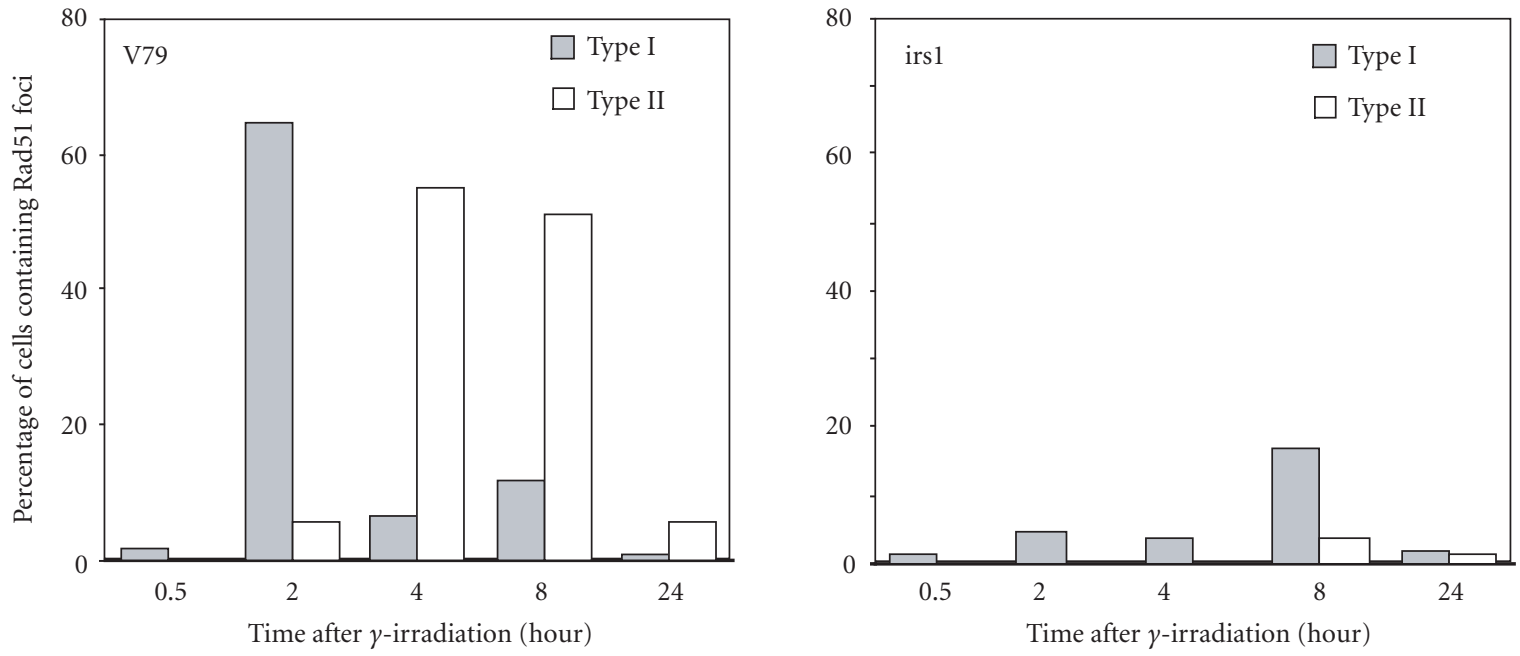

(b)

Figure 5. (a) Hamster nuclei containing type I and type II Rad51 foci (top panels). The correspondent nuclei stained with DAPI are shown in bottom. (b) Percentage of cells containing type I or type II foci in V79 (left) and irs1 (right) cells irradiated with 8 Gy $\gamma$-rays and incubated at $37^{\circ} \mathrm{C}$ for various times.

type II foci ( $>5$ type II foci/cell) became predominant at 4 and 8 hours (Figure 5b). At 8 hours, the fraction of irs 1 cells containing type I (16.8\%) is comparable to that of V79 cells containing only type I foci (11.8\%). But the fraction of cells containing type II foci and the total number of cells containing both types of foci are significantly lower in irs1 cells than those in the wild type (Figure 5b). It should be mentioned that about 10-15\% MMC-treated irs1 cells also contain type I foci after continued exposure to MMC (Figure 2). It seems that the signal for the Rad51 focus formation is normal but the kinetics for the assembly of the foci is much slower in the mutant. Taken together, these results suggest that XRCC2 is an essential factor in the assembly of Rad51.

The assembly of Rad51 proteins at the HRR sites in the presynaptic phase is a critical step for initiating homologous pairing and strand exchange. This process is mediated by a number of accessory factors of Rad51. Following the induction of DSB, the processing of the broken ends by exonucleases results in long tracks of single-stranded DNA (ssDNA) tails. Rad51 polymerizes onto ssDNA to form helical nucleoprotein filament, which is capable of conducting homologous pairing and strand exchange [37]. The biochemical study showed that DNA with single-stranded tails is the preferred substrate for Rad51 in the reaction of homologous pairing [26]. The RPA, which specifically binds to ssDNA, assembles at earlier time than Rad51 to form nuclear foci in irradiated yeast cells [12]. Binding of RPA onto ssDNA promotes the assembly of Rad51, probably by removing the secondary structure in the ssDNA [34]. However, excess RPA competes with Rad51 for binding on ssDNA and suppresses the assembly of Rad51 filament [36]. In an in vitro system, the strand exchange reaction of Rad51 is compromised if RPA is present before the nucleation of Rad51 onto ssDNA, but the reaction proceeds efficiently if RPA is added 
after Rad51 binding to ssDNA [37]. The inhibitory effect of RPA can be overcome by the addition of Rad52 or Rad55Rad57 [28, 37]. Rad54 specifically interacts with established Rad51 nucleoprotein filaments in the synaptic phase to promote homology search on the duplex DNA and heteroduplex DNA formation [33].

The function of XRCC2 and other Rad51 paralogs in the assembly of Rad51-ssDNA is not known at present. Several studies have recently shown that XRCC2 is involved in a multiprotein complex of Rad51 paralogs. Two distinct Rad51 paralog complexes have been identified in human cell extracts, one containing XRCC2, Rad51B, C, and D, and another containing Rad51C and XRCC3 [22, 24, 27, 46]. XRCC2 directly interacts with Rad51D as suggested by a yeast two-hybrid study [30]. Purified Rad51B-C-DXRCC2 and XRCC3-Rad51C complexes preferentially bind to single-stranded, rather than double-stranded, DNA [20, 24, 25]. Significantly, the XRCC3-Rad51C complex forms protein-DNA networks in vitro [24]. These Rad51 paralog complexes also show low level of ssDNA dependent ATPase activities [20, 24, 25]. Interestingly, Rad51D [5] or Rad51C [24] alone also possesses an ssDNA-binding and an ATPase activity. The specificity of binding to singlestranded DNA would be consistent with a role of the Rad51 paralogs in facilitating the formation of Rad51 nucleoprotein filaments. One report suggested that the XRCC3Rad51C heterodimer has homologous paring activity as determined by the D-loop formation between single-stranded and double-stranded oligonucleotides [20]. No interaction between Rad51B-C-D-XRCC2 and Rad51 is observed in cell extracts or in the reaction with purified proteins [22, $25,27,46]$. It is suggested by a yeast two-hybrid study that Rad51 binds to XRCC3 [21]. Immunoprecipitation experiments showed that Rad51 coprecipitated with Rad51CXRCC3 complex in HeLa S3 cell extracts [21, 22]. However, reports from other labs did not show the interaction $[25,27$, 46]. Interestingly, Rad55 interacts with Rad51 in a yeast twohybrid system [16, 18] and purified Rad55 binds to Rad51 in vitro [38], but Rad55-Rad57 is not found to coprecipitate Rad51 in cell extracts [38]. Besides, immunoprecipitation study demonstrated that neither Rad51B-C-D-XRCC2 nor Rad51C-XRCC3 interacts with RPA in human cell extracts (Liu N, unpublished data).

Vertebrate Rad51 paralogs may act as the Rad55-Rad57 complex in terms of promoting the activity of Rad51 at the early stage of HRR, but the function of vertebrate Rad51 paralogs may be diverged from their yeast counterparts. It is not known why there are two Rad51 paralog complexes in vertebrates and how they exert the function together. The Rad51 paralog complexes seem to possess a similar function but neither of them is dispensable, since the disruption of each of the Rad51 paralogs in chicken cells resulted in almost identical phenotypes $[39,40]$. Moreover, Rad55-Rad57 is only required for Rad51 focus formation in meiotic cells, but not required in mitotic yeast cells $[12,13]$, while all of the Rad51 paralogs are necessary for the mitotic Rad51 focus formation. Biochemical studies are currently undertaken to better understand the mediator activity of the Rad51 paralogs in
HRR and the co-operation of Rad51 paralogs with other mediators, such as Rad52, Rad54, BRCA1, and BRCA2.

\section{ACKNOWLEDGMENTS}

I thank Dr Larry Thompson for his encouragement and support during the study. I also thank Dr Randy Legerski for kindly supplying the mouse Rad51 antibody. This work was supported by Grant CA84407-01 from the National Cancer Institute and was performed under the auspices of the US Department of Energy by Lawrence Livermore National Laboratory under contract No W-7405-ENG-48.

\section{REFERENCES}

[1] Baumann P, Benson FE, West SC. Human Rad51 protein promotes ATP-dependent homologous pairing and strand transfer reactions in vitro. Cell. 1996;87(4):757766.

[2] Bhattacharyya A, Ear US, Koller BH, Weichselbaum RR, Bishop DK. The breast cancer susceptibility gene BRCA1 is required for subnuclear assembly of Rad51 and survival following treatment with the DNA cross-linking agent cisplatin. J Biol Chem. 2000;275(31):23899-23903.

[3] Bishop DK. RecA homologs Dmc1 and Rad51 interact to form multiple nuclear complexes prior to meiotic chromosome synapsis. Cell. 1994;79(6):1081-1092.

[4] Bishop DK, Ear U, Bhattacharyya A. Xrcc3 is required for assembly of Rad51 complexes in vivo. J Biol Chem. 1998;273(34):21482-21488.

[5] Braybrooke JP, Spink KG, Thacker J, Hickson ID. The RAD51 family member, RAD51L3, is a DNA-stimulated ATPase that forms a complex with XRCC2.J Biol Chem. 2000;275(37):29100-29106.

[6] Caldecott K, Jeggo P. Cross-sensitivity of gamma-raysensitive hamster mutants to cross-linking agents. $\mathrm{Mu}$ tat Res. 1991;255(2):111-121.

[7] Cartwright R, Tambini CE, Simpson PJ, Thacker J. The XRCC2 DNA repair gene from human and mouse encodes a novel member of the recA/RAD51 family. $\mathrm{Nu}$ cleic Acids Res. 1998;26(13):3084-3089.

[8] Chen J, Silver DP, Walpita D, et al. Stable interaction between the products of the BRCA1 and BRCA2 tumor suppressor genes in mitotic and meiotic cells. Mol Cell. 1998;2(3):317-328.

[9] Cui X, Brenneman M, Meyne J, Oshimura M, Goodwin $\mathrm{EH}$, Chen DJ. The XRCC2 and XRCC3 repair genes are required for chromosome stability in mammalian cells. Mutat Res. 1999;434(2):75-88.

[10] De Silva IU, McHugh PJ, Clingen PH, Hartley JA. Defining the roles of nucleotide excision repair and recombination in the repair of DNA interstrand cross-links in mammalian cells. Mol Cell Biol. 2000;20(21):7980-7990.

[11] Fuller LF, Painter RB. A Chinese hamster ovary cell line hypersensitive to ionizing radiation and deficient in repair replication. Mutat Res. 1988;193(2):109-121. 
[12] Gasior SL, Olivares H, Ear U, Hari DM, Weichselbaum R, Bishop DK. Assembly of RecA-like recombinases: distinct roles for mediator proteins in mitosis and meiosis. Proc Natl Acad Sci USA. 2001;98(15):84118418.

[13] Gasior SL, Wong AK, Kora Y, Shinohara A, Bishop DK. Rad52 associates with RPA and functions with Rad55 and Rad57 to assemble meiotic recombination complexes. Genes Dev 1998;12(14):2208-2221.

[14] Gupta RC, Bazemore LR, Golub EI, Radding CM. Activities of human recombination protein Rad51. Proc Natl Acad Sci USA. 1997;94(2):463-468.

[15] Haaf T, Golub EI, Reddy G, Radding CM, Ward DC. Nuclear foci of mammalian Rad51 recombination protein in somatic cells after DNA damage and its localization in synaptonemal complexes. Proc Natl Acad Sci USA. 1995;92(6):2298-2302.

[16] Hays SL, Firmenich AA, Berg P. Complex formation in yeast double-strand break repair: participation of Rad51, Rad52, Rad55, and Rad57 proteins. Proc Natl Acad Sci USA. 1995;92(15):6925-6929.

[17] Johnson RD, Liu N, Jasin M. Mammalian XRCC2 promotes the repair of DNA double-strand breaks by homologous recombination. Nature. 1999;401(6751):397-399.

[18] Johnson RD, Symington LS. Functional differences and interactions among the putative RecA homologs Rad51, Rad55, and Rad57. Mol Cell Biol. 1995;15(9):48434850 .

[19] Jones NJ, Cox R, Thacker J. Isolation and crosssensitivity of X-ray-sensitive mutants of V79-4 hamster cells. Mutat Res. 1987;183(3):279-286.

[20] Kurumizaka H, Ikawa S, Nakada M, et al. Homologouspairing activity of the human DNA-repair proteins Xrcc3.Rad51C. Proc Natl Acad Sci USA. 2001;98(10):5538-5543.

[21] Liu N, Lamerdin JE, Tebbs RS, et al. XRCC2 and XRCC3, new human Rad51-family members, promote chromosome stability and protect against DNA crosslinks and other damages. Mol Cell. 1998;1(6):783-793.

[22] Liu N, Schild D, Thelen MP, Thompson LH. Involvement of Rad51C in two distinct protein complexes of Rad51 paralogs in human cells. Nucleic Acids Res. 2002;30(4):1009-1015.

[23] Liu Y, Maizels N. Coordinated response of mammalian Rad51 and Rad52 to DNA damage. EMBO Rep. 2000;1(1):85-90.

[24] Masson JY, Stasiak AZ, Stasiak A, Benson FE, West SC. Complex formation by the human RAD51C and XRCC3 recombination repair proteins. Proc Natl Acad Sci USA. 2001;98(15):8440-8446.

[25] Masson JY, Tarsounas MC, Stasiak AZ, et al. Identification and purification of two distinct complexes containing the five RAD51 paralogs. Genes Dev. 2001;15(24):3296-3307.

[26] Mazin AV, Zaitseva E, Sung P, Kowalczykowski SC. Tailed duplex DNA is the preferred substrate for Rad51 protein-mediated homologous pairing. EMBO J.
2000;19(5):1148-1156.

[27] Miller KA, Yoshikawa DM, McConnell IR, Clark R, Schild D, Albala JS. RAD51C interacts with RAD51B and is central to a larger protein complex in vivo exclusive of RAD51. J Biol Chem. 2002;277(10):8406-8411.

[28] New JH, Sugiyama T, Zaitseva E, Kowalczykowski SC. Rad52 protein stimulates DNA strand exchange by Rad51 and replication protein A. Nature. 1998;391(6665):407-410.

[29] O’Regan P, Wilson C, Townsend S, Thacker J. XRCC2 is a nuclear RAD51-like protein required for damagedependent RAD51 focus formation without the need for ATP binding. J Biol Chem. 2001;276(25):2214822153.

[30] Schild D, Lio Y, Collins DW, Tsomondo T, Chen DJ. Evidence for simultaneous protein interactions between human Rad51 paralogs. J Biol Chem. 2000;275(22):16443-16449.

[31] Scully R, Chen J, Ochs RL, et al. Dynamic changes of BRCA1 subnuclear location and phosphorylation state are initiated by DNA damage. Cell. 1997;90(3):425-435.

[32] Shen Z, Cloud KG, Chen DJ, Park MS. Specific interactions between the human RAD51 and RAD52 proteins. J Biol Chem. 1996;271(1):148-152.

[33] Solinger JA, Lutz G, Sugiyama T, Kowalczykowski SC, Heyer WD. Rad54 protein stimulates heteroduplex DNA formation in the synaptic phase of DNA strand exchange via specific interactions with the presynaptic Rad51 nucleoprotein filament. J Mol Biol. 2001;307(5):1207-1221.

[34] Sugiyama T, Zaitseva EM, Kowalczykowski SC. A single-stranded DNA-binding protein is needed for efficient presynaptic complex formation by the Saccharomyces cerevisiae Rad51 protein. J Biol Chem. 1997;272(12):7940-7945.

[35] Sung P. Catalysis of ATP-dependent homologous DNA pairing and strand exchange by yeast RAD51 protein. Science. 1994;265(5176):1241-1243.

[36] Sung P. Yeast Rad55 and Rad57 proteins form a heterodimer that functions with replication protein A to promote DNA strand exchange by Rad51 recombinase. Genes Dev. 1997;11(9):1111-1121.

[37] Sung P, Robberson DL. DNA strand exchange mediated by a RAD51-ssDNA nucleoprotein filament with polarity opposite to that of RecA. Cell. 1995;82(3):453-461.

[38] Sung P, Trujillo KM, Van Komen S. Recombination factors of Saccharomyces cerevisiae. Mutat Res. 2000;451(1-2):257-275.

[39] Takata M, Sasaki MS, Sonoda E, et al. The Rad51 para$\log$ Rad51B promotes homologous recombinational repair. Mol Cell Biol. 2000;20(17):6476-6482.

[40] Takata M, Sasaki MS, Tachiiri S, et al. Chromosome instability and defective recombinational repair in knockout mutants of the five Rad51 paralogs. Mol Cell Biol. 2001;21(8):2858-2866.

[41] Tan TL, Essers J, Citterio E, et al. Mouse Rad54 affects DNA conformation and DNA-damage-induced Rad51 foci formation. Curr Biol. 1999;9(6):325-328. 
[42] Tashiro S, Kotomura N, Shinohara A, Tanaka K, Ueda K, Kamada N. S phase specific formation of the human Rad51 protein nuclear foci in lymphocytes. Oncogene. 1996;12(10):2165-2170.

[43] Tebbs RS, Zhao Y, Tucker JD, et al. Correction of chromosomal instability and sensitivity to diverse mutagens by a cloned cDNA of the XRCC3 DNA repair gene. Proc Natl Acad Sci USA 1995;92(14):6354-6358.

[44] Thompson LH, Schild D. Homologous recombinational repair of DNA ensures mammalian chromosome stability. Mutat Res. 2001;477(1-2):131-153.

[45] Tucker JD, Jones NJ, Allen NA, Minkler JL, Thompson LH, Carrano AV. Cytogenetic characterization of the ionizing radiation-sensitive Chinese hamster mutant irs1. Mutat Res. 1991;254(2):143-152.

[46] Wiese C, Collins DW, Albala JS, Thompson LH, Kronenberg A, Schild D. Interactions involving the Rad51 paralogs Rad51C and XRCC3 in human cells. Nucleic Acids Res. 2002;30(4):1001-1008.

[47] Yuan SS, Lee SY, Chen G, Song M, Tomlinson GE, Lee EY. BRCA2 is required for ionizing radiationinduced assembly of Rad51 complex in vivo. Cancer Res. 1999;59(15):3547-3551.

\footnotetext{
* E-mail: liu3@llnl.gov

Fax: +1 925422 2282; Tel: +1 9254225630
} 

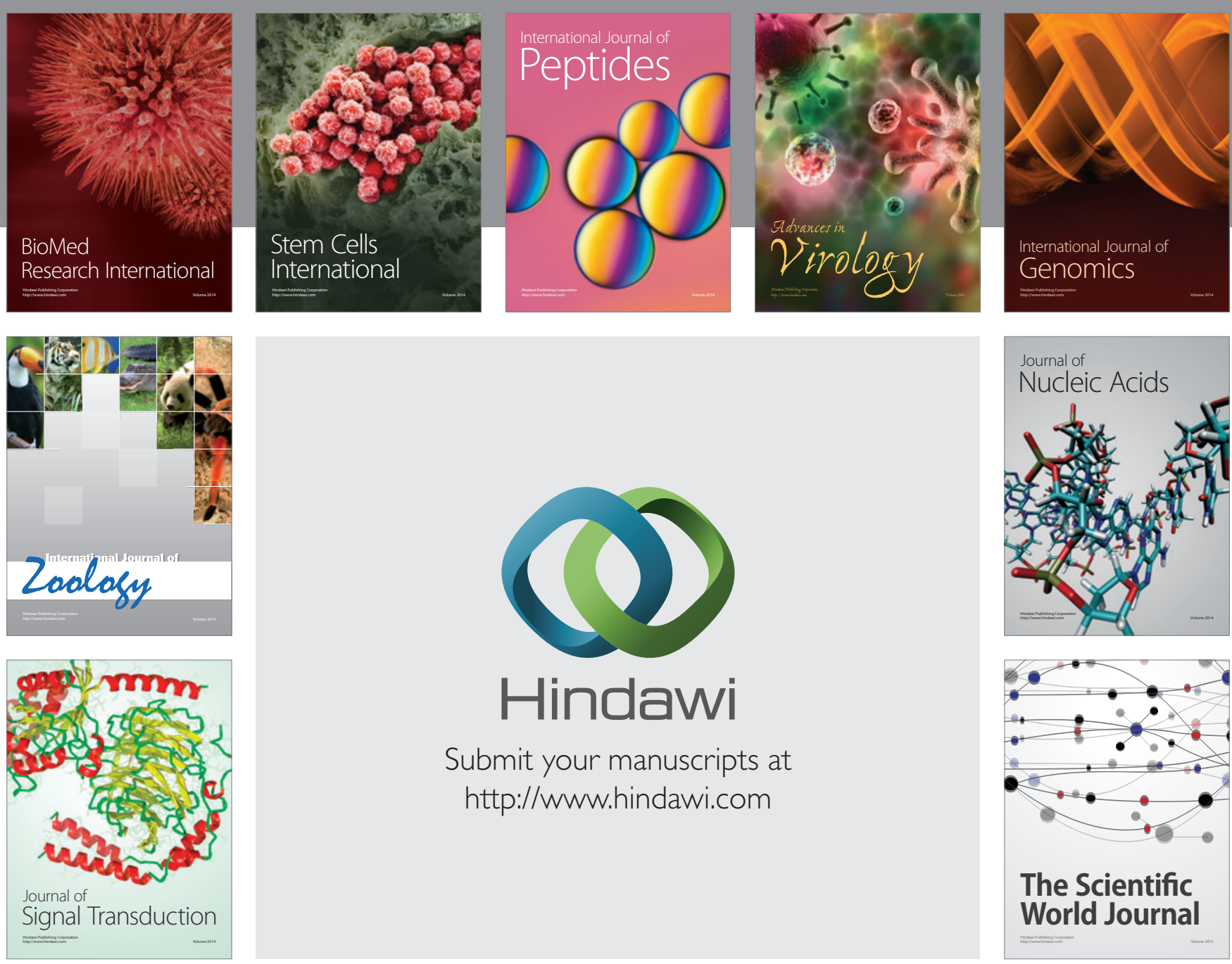

Submit your manuscripts at

http://www.hindawi.com
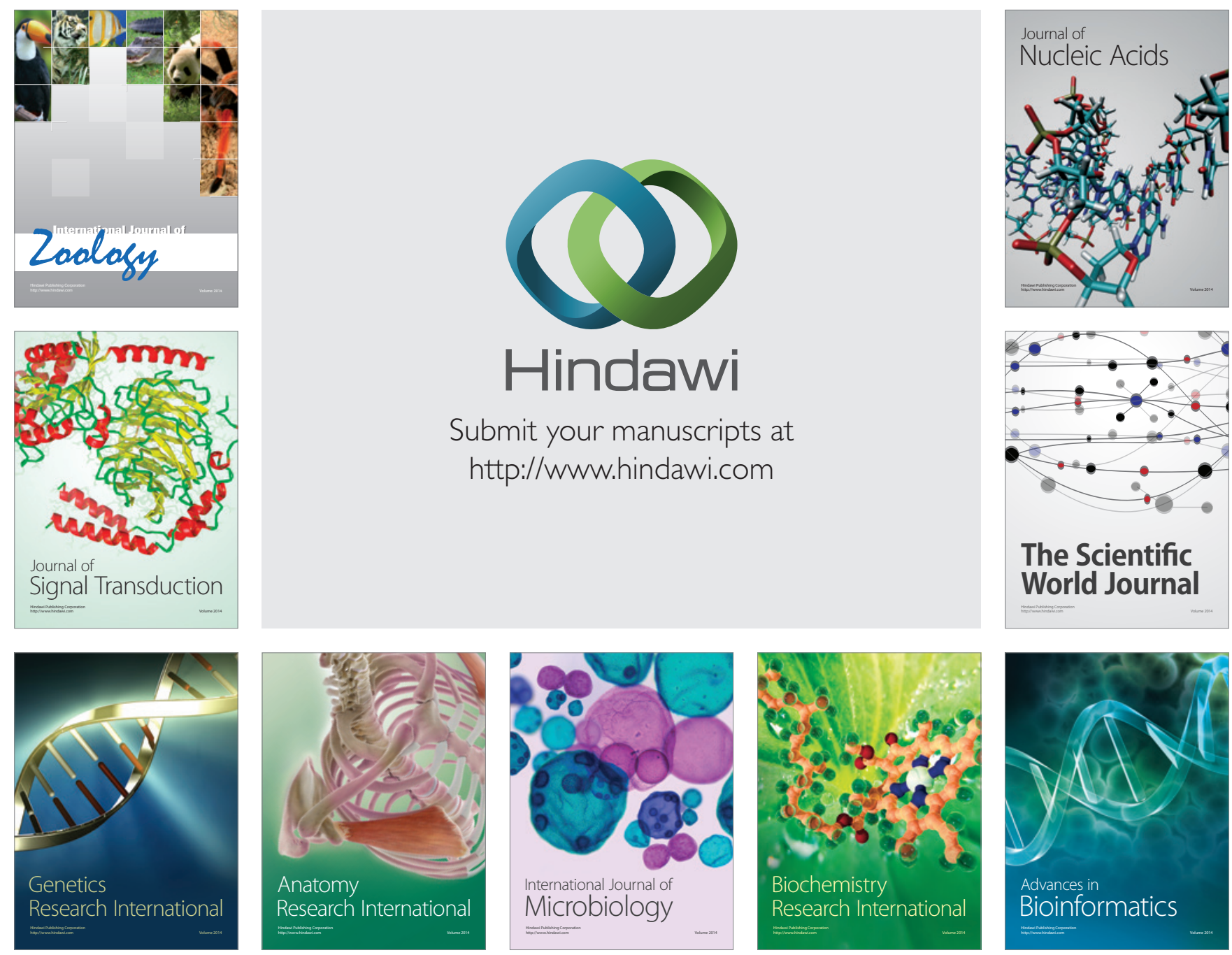

The Scientific World Journal
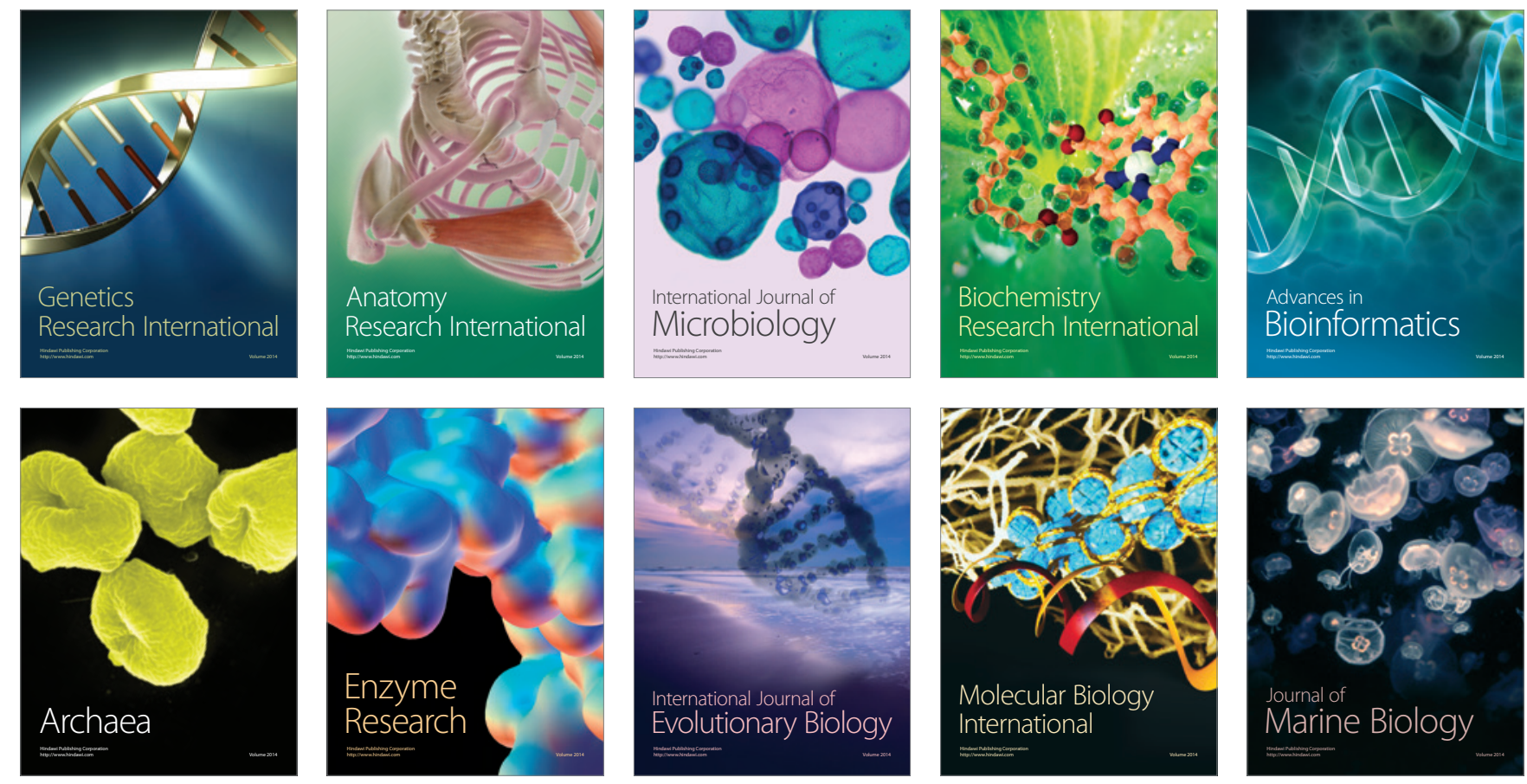